\title{
Endogenous endophthalmitis: experience from the south of peninsular Malaysia
}

Norfariza Ngah ${ }^{1,2}$

${ }^{1}$ Ophthalmology Service, Ministry of Health, Kuala Lumpur, Malaysia; ${ }^{2}$ Department of Ophthalmology, Hospital Shah Alam, Persiaran Kayangan, Shah Alam, Selangor, Malaysia

Although endogenous endophthalmitis is a relatively rare intraocular infection, a higher incidence has been reported in high-risk patients. This potentially blinding ocular infection results from hematogenous spread of organisms from a remote primary source. Chronically ill or debilitated patients as well as those who underwent any invasive procedures are especially at risk. Ophthalmologists and medical practitioners must have a high index of suspicion in high-risk cases to allow for prompt diagnosis and treatment.

Many etiologic organisms, namely gram-positive, gram-negative, and fungal have been reported to cause endogenous endophthalmitis. Different parts of the world show different types of common organisms, with Staphylococcus aureus and Streptococcal pneumoniae being more common in Europe and North America and Klebsiella pneumoniae more common in East Asia. Candida albicans, on the other hand, is the most common yeast and Aspergillus sp. is the most common mold. ${ }^{1}$

Endogenous endophthalmitis has no age or sexual predilection. The right eye is involved twice as often as the left eye because of the more proximal and direct blood flow to the right carotid artery. Bilateral involvement occurs in up to $25 \%$ of cases. ${ }^{2}$

Prompt administration of antibiotic therapy is key in the acute management of endogenous endophthalmitis. Surgical intervention is generally recommended for patients infected with more virulent organisms, visual acuity of 3/60 or less, or severe vitreous involvement. The outcome of posterior diffuse endophthalmitis or panophthalmitis is frequently blindness, regardless of treatment measures. ${ }^{3}$

In the case series reported in this issue by Hayatulrizal et al., ${ }^{4}$ negative microbial culture was seen in 11 eyes (57.9\%) and were treated with empirical systemic and intravitreal antibiotics. Despite aggressive treatment, the visual outcomes were

Correspondence: Norfariza Ngah, Head of Department, Department of Ophthalmology, Hospital Shah Alam, Persiaran Kayangan, Shah Alam, Selangor, Malaysia.

E-mail: drfarizangah@gmail.com 
rather poor, with nearly $75 \%$ of patients showing no improvement or worsening of vision. Vitrectomy was performed only in eight eyes.

The outcome of endogenous endophthalmitis is often disappointing. The three main factors that contribute to poor prognosis include more virulent organisms, compromised host conditions, and delayed diagnosis. Therefore, a high degree of suspicion is necessary to make an early diagnosis of endogenous endophthalmitis. ${ }^{5}$

\section{References}

1. Lingappan A, Wykoff CC, Albini TA, et al. Endogenous fungal endophthalmitis: causative organisms, management strategies, and visual acuity outcomes. Am J Ophthalmol. 2012:253(1):162-166.

2. Greenwald MJ, Wohl LG, Sell CH. Metastatic bacterial endophthalmitis: a contemporary reappraisal. Surv Ophthalmol. 1986;31:81-101.

3. Chee SP, Jap A. Endogenous endophthalmitis. Curr Opin Ophthalmol. 2001;12:464-470.

4. Hayatulrizal M, LK Phang, FM Vendargon. A five-year retrospective hospital-based study of endogenous endophthalmitis in south Malaysia. Malaysian Journal of Ophthalmology. 2020;2(1):18-26.

5. Okada AA, Johnson RP, Liles WC, D’Amico DJ, Baker AS. Endogenous bacterial endophthalmitis. Report of a ten-year retrospective study. Ophthalmology. 1994;101:832-838. 